\title{
Sistematización en el proceso de evaluación y terapia del pólipo de cuerda vocal. Encuesta a médicos Otorrinolaringólogos Chilenos.
}

\section{Assessment and therapy process systematization of vocal fold polyps. A survey to chilean otorhynolaryngologists}

Loreto Nercelles C. Fonoaudióloga

Escuela de Fonoaudiología Universidad Andrés Bello

\section{Daniela Fernández C. Fonoaudióloga \\ Lucía Retamales V. Fonoaudióloga}

\section{Francisca Vergara S.} Fonoaudióloga

\section{RESUMEN}

Esta investigación tiene por propósito describir las indicaciones terapéuticas, prescritas por médicos otorrinolaringólogos a pacientes diagnosticados con pólipo cordal. Se describen las etapas de evaluación, reposo vocal e indicaciones terapéuticas. El estudio intenta develar la presencia de una sistematización en el proceso anterior.

Dicha información es recabada a partir de una encuesta vía mail aplicada a 29 médicos pertenecientes a la Sociedad Chilena de Otorrinolaringología ( $N=205)$. Los datos obtenidos permiten concluir que no existe un estándar en las indicaciones que se preguntan en la encuesta. Sin embargo, el total de médicos consultados manifiesta que sería útil la elaboración de protocolos para la evaluación y tratamiento de los pacientes con alteración vocal.

Palabras claves: voz, pólipo cuerda vocal, evaluación.

\section{ABSTRACT}

This research is aimed at describing the therapeutic indications, prescribed by otorhynolaryngologists polyp patients diagnosed with tailpiece, including assessment, voice rest and therapeutic indications. The study attempts to reveal the presence of systematization in the previous process. This information is collected from a survey via e-mail applied to 29 physicians belonging to the Chilean Society of Otolaryngology ( $n=205$ ). The data obtained indicate that there is no standard in the information asked for in the survey. However, the total number of doctors surveyed stated that the development of protocols for assessment and treatment of patients with vocal alteration would be useful.

Key words: voice, vocal cord polyps, assessment.
Contacto con el autor: Loreto Nercelles C. Escuela de Fonoaudiología Universidad Andrés Bello Fernández Concha 700 Santiago - Chile Tel: (56 2) 6618262

Correo-e: loreto.nercelles@unab.cl 


\section{Introducción}

El proceso de evaluación y terapia de los trastornos de la voz se inicia con la evaluación realizada por el médico otorrinolaringólogo, quien debe valorar mediante diferentes técnicas exploratorias la indemnidad anatómica y funcional de la laringe. Además, según la patología o trastorno diagnosticado, este profesional es el encargado de derivar a terapia fonoaudiológica, indicar tratamiento farmacológico o quirúrgico y proporcionar pautas sobre higiene vocal ${ }^{1,2}$.

Un buen diagnóstico médico debería considerar características como reproductibilidad, validez y valor predictivo. Las debilidades en cualquiera de las anteriores pueden debilitar la exactitud de la evaluación y poner en peligro las consecuentes decisiones para el tratamiento ${ }^{3}$. Además, el éxito de la terapia puede ser altamente dependiente de cómo se diagnostica e identifica la patología vocal ${ }^{2}$.

En el marco de este continuo, existen diversos estudios de exploración en los que a través de una serie de cuestionarios o encuestas dirigidas al equipo rehabilitador, se evidencia una falta de sistematización en el proceso de evaluación de la $\mathrm{voz}^{4,5}$. El equipo de Rodríguez-Parra (2006) realizó un sondeo en diferentes hospitales españoles, con el objetivo de conocer la variabilidad en los procedimientos tanto de evaluación como rehabilitación de los trastornos de voz. Para lo anterior, confeccionaron una encuesta con temas concernientes al grado de colaboración interdisciplinar en el manejo de pacientes disfónicos, utilización de un protocolo sistemático de evaluación y tratamiento vocal, tipo de exploración más frecuentemente utilizada, frecuencia de los seguimientos realizados, disposición de un servicio de laboratorio de voz y porcentaje de éxito atribuido a los distintos tratamientos.

Por su parte, Behrman (2004) ${ }^{6}$ encuestó a terapeutas pertenecientes a la ASHA (American Speech Language Hearing Association) sobre las herramientas y prácticas de evaluación utilizadas en disfonías musculotensionales. Se concluyó que la única práctica común era la medición de la calidad vocal y la autovaloración de la disfonía por parte del paciente. Las otras prácticas tales como medir postura, cambios en la emisión, estroboscopía, análisis fonético acústico, medición aerodinámica o Electroglotografía (EGG) no siempre se realizaban.

Una de las dificultades en la sistematización del proceso, es la existencia de una variedad de herramientas para diagnosticar una voz como disfónica6. Por lo anterior, es conveniente revisar qué dimensiones hay que evaluar, con qué pruebas, qué valores son patológicos, hasta llegar a una evaluación protocolizada ${ }^{7,8}$. Dejonckere y colaboradores $(2001)^{9}$ elaboraron un protocolo básico de evaluación y manejo de la patología vocal, especialmente para las que requieren tratamiento fonoquirúrgico. En este se desarrolla la evaluación perceptual, estroboscópica, acústica, aerodinámica y la propia del paciente. Además, señalan que es una necesidad que el equipo rehabilitador tenga similares criterios y trabaje en forma colaborativa. 
La presente investigación pretende describir la presencia de sistematización en las indicaciones relacionadas con evaluación, reposo vocal y terapia fonoaudiológica entregadas por los médicos otorrinolaringólogos pertenecientes a la Sociedad Chilena de Otorrinolaringología, en relación a la patología del pólipo de cuerda vocal. Cabe destacar que se eligió esta patología por ser una de las lesiones más comunes en las series quirúrgicas ${ }^{10}$.

\section{Metodología}

La muestra correspondió a un total de 29 otorrinolaringólogos pertenecientes a la Sociedad Chilena de Otorrinolaringología, todos ellos especialistas que han tenido su formación avalada por la ASOFAMECH (Asociación de Facultades de Medicina de Chile) o bien han sido aprobados por el CONACEM (Comisión Nacional de Acreditación de Especialidades Médicas)

El rango de años de ejercicio de la profesión fluctuó entre 9 y 33 años de experiencia, con una media de 21 años. El mayor porcentaje de ellos se desempeña profesionalmente en instituciones tanto públicas como privadas (66\%), un $24 \%$ en institución privada y un $10 \%$ exclusivamente en institución pública. Además, tienen una media de 40 microcirugías laríngeas efectuadas en los últimos 5 años.

La metodología utilizada para la recolección de los datos consistió en el envío mediante correo electrónico de una encuesta tipo cuestionario al mail de la sociedad Chilena de otorrinolaringología que difundió la información a sus respectivos socios. Los médicos interesados en participar enviaron sus respuestas a un mail determinado por los investigadores, posteriormente se efectuó el análisis de los resultados sobre la muestra obtenida.

El instrumento (anexo 1) incluyó preguntas de tipo abierto y cerrado, considerando preguntas dicotómicas y de opción múltiple. El contenido se dividió en tres ítems principales. En el primero, preguntas relacionadas al ejercicio de la especialidad y procedimientos quirúrgicos realizados. En el segundo, características de la evaluación, preguntas estaban destinadas a obtener información sobre la utilización de protocolos de evaluación de pacientes disfónicos, técnicas exploratorias y procedimientos empleados. Finalmente, en el tercer ítem se abordó de forma directa, temáticas dirigidas al conocimiento sobre la intervención de los pacientes, indicaciones pre y post-quirúrgicas generales, controles efectuados y complicaciones asociadas.

Por otra parte, se anexó al cuestionario un consentimiento informado señalando el objetivo de la investigación e indicando el carácter anónimo de las respuestas obtenidas a partir de este. Se enfatizó en que la información recolectada sería utilizada solo para fines del estudio.

\section{Resultados}

Del total de la muestra, el $65,5 \%$ de los encuestados utiliza un protocolo de evaluación de pacientes disfónicos. De este grupo los mayores porcentajes se distribuyen equitativamente entre protocolos de la unidad o centro y un $36,8 \%$ 
protocolos de procedencia extranjera. El 26, 3\% corresponde a protocolo de elaboración propia.

En el gráfico 1 , se muestran los resultados sobre las técnicas exploratorias utilizadas en la evaluación de pacientes disfónicos. Se obtiene que el mayor porcentaje lo obtiene la nasofibroscopía con un $100 \%$, seguida por la laringoscopía indirecta con un $72 \%$. En oposición a éstos, las técnicas menos utilizadas corresponden a la psicoacústica (tonotimbre-intensidad) y la medición aerodinámica con un $17 \%$ y $7 \%$ respectivamente.

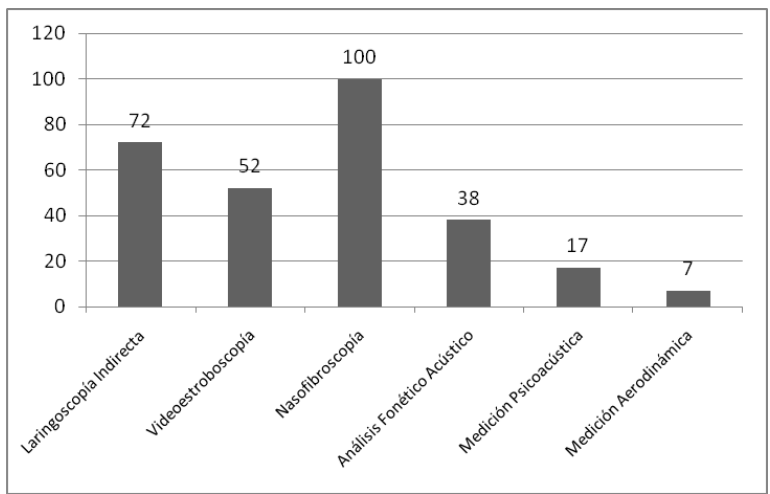

Gráfico 1. Técnicas exploración evaluación.

Acerca de las indicaciones iniciales o inmediatas que el ORL entrega al paciente diagnosticado con pólipo de la cuerda vocal, se encuentra la indicación de higiene vocal y microcirugía laríngea con un 59\%, seguida por el reposo vocal relativo con un $55 \%$. Las indicaciones menos prescritas corresponden a reposo vocal absoluto y medicamentos con un $0 \%$ y $35 \%$, respectivamente. Llama la atención que dentro de las respuestas, la terapia fonoaudiológica prequirúrgica ocupe el cuarto lugar dentro de las indicaciones prescritas por el médico (52\%) (gráfico 2).

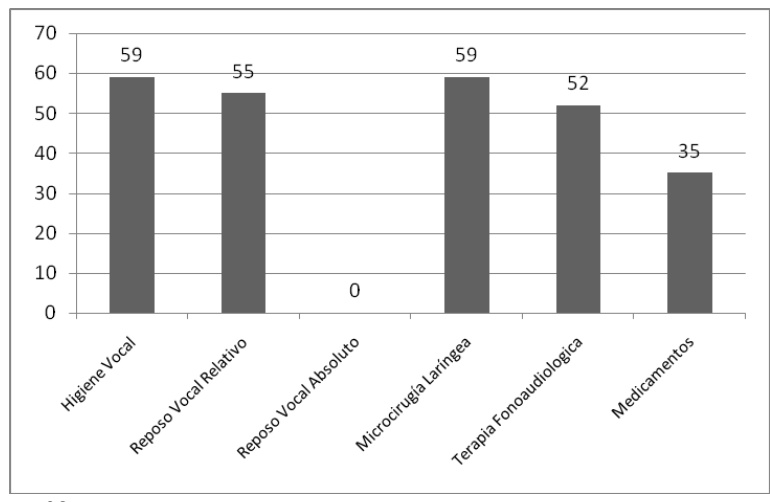

Gráfico 2. Indicaciones iniciales.

También, se estudió el porcentaje de médicos ORL que prescribían de manera conjugada reposo vocal relativo, pautas de higiene, terapia fonoaudiológica y microcirugía laríngea como indicación inicial, observándose que solo un $24 \%$ de la muestra recomienda seguir las cuatro indicaciones.

En relación al tipo de cirugía utilizada para la extirpación del pólipo de cuerda vocal, el 96,6\% usa microcirugía laríngea clásica y el 10,3 \% microcirugía con láser de $\mathrm{CO} 2$.

Con respecto a las indicaciones postquirúrgicas más recurrentes que el ORL entrega al paciente, se encuentran terapia fonoaudiológica (97\%), reposo vocal relativo (90\%), tratamiento farmacológico (86\%), reposo vocal absoluto (79\%) y pautas de higiene vocal (79\%) (gráfico 3).

En relación al reposo vocal absoluto como recomendación post-quirúrgica que el ORL entrega al paciente, se obtiene que dentro de los mayores porcentajes se encuentra la categoría de 4 a 7 días con un $35 \%$, seguido por 1 a 3 días con un $31 \%$. Se evidencia que la adherencia a prescribir reposo vocal 
absoluto es menor que al relativo y se distribuyen de manera más similar que este último.

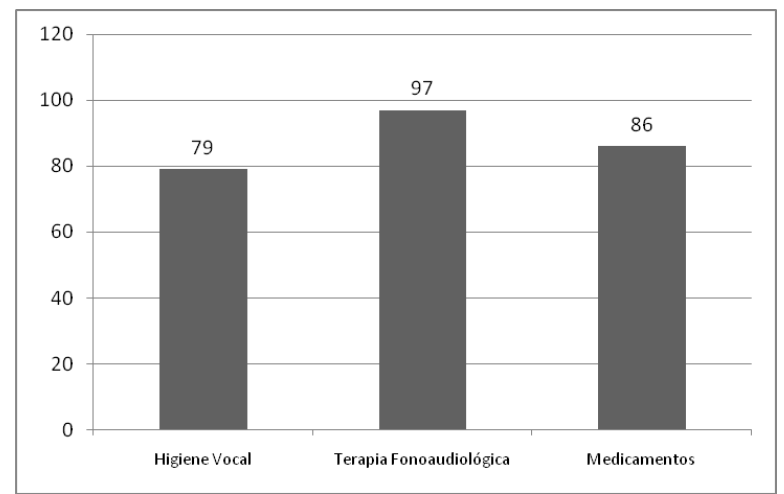

Gráfico 3. Indicaciones postquirúrgicas ORL.

En cuanto al reposo vocal relativo postquirúrgico, los mayores porcentajes son las categorías de 4 a 7 días con un $52 \%$, seguido por 8 a14 días con un 31\%. El 93,1\% de los encuestados considera que el tipo de reposo depende del uso de la voz, un $27,6 \%$ de patologías previas y un $20,7 \%$ del tamaño del pólipo.

En la tabla 1 se resumen las conductas que el médico ORL prohíbe antes o después de la cirugía. Se evidencia con claridad que las conductas con más altos porcentajes de prohibición corresponden a consumo de tabaco, hablar a intensidad alta y cantar. Además, se aprecia un considerable aumento en todas las conductas durante el período postquirúrgico, en especial toser o carraspear $(69 \% \mathrm{v} / \mathrm{s}$ $34,5 \%)$, llorar $(20,7 \%$ v/s $10,3 \%)$ y realizar esfuerzos físicos (62,1\% v/s 24,1\%).

En relación a los porcentajes de respuestas obtenidos sobre la periodicidad de control de la evolución del paciente, un 86\% manifiesta controlar a la semana, seguido por un $52 \%$ al mes. Se observa que un alto porcentaje de los encuestados practica una metodología de revisión o reevaluación a la semana y al mes.

Asimismo, de acuerdo a los porcentajes obtenidos se puede visualizar que los médicos realizan más de un control al paciente.

Con respecto a las técnicas exploratorias que los médicos utilizan en el control de los pacientes, el mayor porcentaje lo obtiene la nasofibroscopía con un $100 \%$, seguida por la estroboscopía con un 35\%. En oposición a estos, las técnicas menos utilizadas, al igual que en el proceso de evaluación, corresponden a laringoscopía indirecta 17\%, psicoacústica (tonotimbre-intensidad) y medición aerodinámica con un $14 \%$ y $7 \%$, respectivamente.

Se evidencia que del total de la muestra, el $88,9 \%$ de los médicos señala que sus pacientes recidivan en un rango de $0-25 \%$. Además, el $100 \%$ señala que resultaría útil la elaboración de un protocolo de evaluación y tratamiento vocal sistematizado. 
Tabla 1. Características principales de las afasias.

\begin{tabular}{|c|c|c|c|c|}
\hline \multirow{2}{*}{ CONDUCTAS } & \multicolumn{2}{|c|}{ PRECIRUGIA } & \multicolumn{2}{|c|}{ POSTCIRUGIA } \\
\hline & $\mathbf{n}$ & $\%$ & $\mathbf{n}$ & $\%$ \\
\hline Consumir tabaco & 26 & $89,7 \%$ & 29 & $100 \%$ \\
\hline Consumir alcohol & 19 & $65,5 \%$ & 20 & $69 \%$ \\
\hline Consumir café & 15 & $51,7 \%$ & 18 & $62,1 \%$ \\
\hline Alimentos irritantes o muy condimentados & 15 & $51,7 \%$ & 20 & $69 \%$ \\
\hline Consumir alimentos a temperatura muy fría/ caliente & 7 & $24,1 \%$ & 11 & $37,9 \%$ \\
\hline Toser o carraspear & 10 & $34,5 \%$ & 20 & $69 \%$ \\
\hline Hacer gárgaras & 5 & $17,2 \%$ & 7 & $24,1 \%$ \\
\hline Hablar a intensidad alta & 22 & $75,9 \%$ & 29 & $100 \%$ \\
\hline Cantar & 21 & $72,4 \%$ & 29 & $100 \%$ \\
\hline Hablar en voz susurrada & 15 & $51,7 \%$ & 18 & $62,1 \%$ \\
\hline Llorar & 3 & $10,3 \%$ & 6 & $20,7 \%$ \\
\hline Realizar esfuerzos físicos & 7 & $24,1 \%$ & 18 & $62,1 \%$ \\
\hline Hablar excesivamente por teléfono & 18 & $62,1 \%$ & 22 & $75,9 \%$ \\
\hline \multicolumn{5}{|l|}{ Otras: } \\
\hline Gritar & 1 & $3,4 \%$ & 1 & $3,4 \%$ \\
\hline Suspender aspirina & 1 & $3,4 \%$ & 1 & $3,4 \%$ \\
\hline
\end{tabular}

\section{Discusión}

Los datos obtenidos permiten concluir que no existe un estándar de indicaciones sobre la evaluación y terapia para pacientes adultos diagnosticados con pólipo de cuerda vocal, que incluya recomendaciones de reposo vocal, terapia fonoaudiológica, pautas de higiene vocal $y$ microcirugía laríngea.

Con respecto a esto último, en lo que concierne a las técnicas exploratorias utilizadas por los médicos otorrinolaringólogos en la evaluación de pacientes disfónicos en el período pre-quirúrgico, se observó que la técnica más ampliamente utilizada era la nasofibroscopía (100\%), seguida por la laringoscopía indirecta (72\%). En oposición a estas, las menos utilizadas resultaron ser la psicoacústica (17\%) y la medición aerodinámica (7\%). Asimismo, los porcentajes de utilización de estas técnicas en los controles post-quirúrgicos son similares, ya que la nasofibroscopía es empleada por el $100 \%$ de los sujetos del estudio, además de presentarse nuevamente bajos porcentajes para las mediciones aerodinámicas (7\%) y psicoacústicas (14\%). Lo descrito concuerda con lo señalado en el estudio realizado por Rodríguez-Parra et al. $(2006)^{8}$, al evidenciar que la gran mayoría de los especialistas continúan basando la exploración vocal en pruebas de exploración médicas y anatómicas (nasofibroscopía, laringoscopía indirecta). Por tanto otorgan menos importancia a otras medidas de carácter funcional con gran valor diagnóstico y pronóstico, como lo son la estroboscopía y medición aerodinámica. De lo anterior se puede inferir que la baja utilización de la estroboscopía podría explicarse por los altos costos que implica su implementación, considerando que una gran mayoría de los médicos ORL se desempeña en el sistema público. Resulta lamentable que no se considere la medición aerodinámica, ya que es primordial para el análisis de aspectos tales como la eficiencia de la fonación y el esfuerzo vocal. Para ello es posible efectuar la evaluación del tiempo máximo de fonación y soplo, así como el cálculo del índice s/z que permite analizar 
el grado de cierre glótico en función de la capacidad respiratoria. Las ventajas de estos procedimientos son el costo mínimo, el uso fácil y la rápida interpretación por parte del especialista.

Por otra parte, la valoración perceptual o psicoacústica de la voz también obtiene un bajo porcentaje de utilización. Núñez, Corte, Senqueiros, Señaris \& Suárez $(2004)^{11}$ señalan que dicha evaluación puede ser sistematizada de forma práctica por el método GIRBAS.

En relación a la implementación de laboratorio de voz en la unidad o centro médico del especialista, se observó que el $58,6 \%$ cuenta con este y que la implementación de equipos de evaluación cualitativa y cuantitativa de la voz es aun incompleta. El beneficio de universalizar esta herramienta de evaluación se traduciría en la posibilidad de realizar una evaluación más completa, otorgando soporte al diagnóstico de las disfonías, además de poder realizar comparaciones de la voz antes y después de la cirugía y del tratamiento fonoaudiológico.

Con respecto a las indicaciones iniciales o inmediatas que son entregadas al paciente, se obtiene que en general los porcentajes individualizados referidos a microcirugía laríngea, terapia fonoaudiológica, higiene vocal y reposo vocal relativo bordean el $50 \%$. Por su parte, entre las indicaciones post-quirúrgicas más recurrentes que el ORL entrega al paciente, se encuentran terapia fonoaudiológica (97\%), reposo vocal relativo (90\%), tratamiento farmacológico (86\%), reposo vocal absoluto y pautas de higiene vocal (ambas con un $79 \%)$. Frente a este hallazgo es relevante destacar la baja adherencia hacia las indicaciones iniciales. Es de interés mencionar que la terapia fonoaudiológica ocupa el cuarto lugar en la etapa pre-quirúrgica dentro de las indicaciones prescritas por los médicos, pese a que la literatura señala que dentro de los tratamientos para el pólipo cordal, la reeducación vocal debe ser complementaria llevándose a cabo en periodos pre y post quirúrgicos.

Le Huche \& Allali (2003) ${ }^{12}$ mencionan que la idea de reeducación preoperatoria a veces encuentra resistencias por parte de médicos preocupados, sobretodo del estado de la laringe, que piensan que el fonoaudiólogo solo podrá comenzar la reeducación de la función vocal tras la rectificación anatómica. Es igualmente errada la actitud de crear una rivalidad entre la cirugía y la reeducación, como si fueran tratamientos equivalentes en su finalidad, dando a entender, por ejemplo, que la cirugía intervendrá en caso de que la reeducación fracase. Es preferible plantear ambos tratamientos, así como otros, asociando racionalmente cada uno con su objetivo y considerándolos en su complementariedad.

Existe diferencia de porcentajes atribuidos a la terapia fonoaudiológica pre y post-operatoria, ya que en la segunda un porcentaje cercano al $100 \%$ manifiesta indicarla. Esto podría demostrar que los médicos reconocen la importancia que tienen las sesiones post-quirúrgicas de reeducación de la voz en la reducción del edema, recuperación de la calidad vocal y el favorecimiento de los nuevos ajustes en la producción vocal.

$\begin{array}{rcr}\text { Frente a la indicación de } & \text { terapia } \\ \text { fonoaudiológica } & \text { se evidenció que existe }\end{array}$


homogeneidad solo en el período post-quirúrgico. En el caso del reposo vocal absoluto su indicación en una fase inicial es uniforme, debido a que la totalidad de los encuestados prescinde de él. Esto no ocurre en la etapa post-operatoria, pues las respuestas de los médicos en lo que respecta a la duración del reposo, no mostraron una preponderancia significativa. El reposo vocal relativo como indicación inicial resulta ser heterogéneo, ya que no existe una mayoría porcentual que manifieste una adherencia o rechazo hacia este. En relación a la prescripción de pautas de higiene vocal, se obtiene una divergencia en períodos pre y post-quirúrgicos.

Las respuestas relacionadas con el trabajo colaborativo entre médico otorrinolaringólogo y fonoaudiólogo especialista en el área de voz, demuestran una intervención del paciente desde ambas especialidades. Esto revela la importancia que los médicos otorrinolaringólogos le asignarían a la participación del terapeuta de voz, en el desarrollo de un trabajo multiprofesional.

Estas conclusiones contribuyen a dilucidar la situación actual en el ámbito de la otorrinolaringología, respecto de ciertas características del ejercicio profesional de los médicos, que hasta ahora eran desconocidos para el área de la Fonoaudiología. La información recabada podría contribuir en una mejor comunicación y trabajo colaborativo de ambas disciplinas, para el beneficio integral de la persona que padece de una afección vocal. 


\section{Referencias}

1. Casado, J. y Adrián, J. (2002). La Evaluación Clínica de la Voz. Fundamentos Médicos y Logopédicos. (1ae ed.). Málaga: Aljibe.

2. Boone, D. \& Mc Farlane, C. (2000).The voice and voice therapy, United States: Allyn and Bacon.

3. Biddler, A.; Watson, L.; Hooper, C.; Lohr, K. \& Sutton, S. (2002). "Criteria for determining disability in speechlanguage disorders. Agency for Healthcare Research and Quality Evidence Report, Number 52.

4. Larson, G. \& Mueller, P. (1991). Voice therapy practices and techniques: a survey of voice clinicians. Journal of Communication Disorders, 25,4: 251-260.

5. Dunnet, C., MacKenzie, K., Sellars, G., Robinson K. \& Wilson, J. (1997) Voice therapy for dysphonia. still more art than science?. European Journal of Disorders Communication, Vol 32: 333-343.

6. Behrman, A. (2005) Common Practices of Voice Therapists in the Evaluation of Patients, Journal of Voice, Vol 19: 454-469.

7. Hirano, M. (1989). Objective evaluation of the human voice: clinical aspects, Folia Foniatrica, 41: 879- 944.

8. Rodríguez-Parra, M., Casado, J., Adrián, J. y Buiza, J. (2006). Estado actual de los Servicios ORL españoles. Heterogeneidad en el manejo de los problemas de voz. Acta de Otorrinolaringología España. España [En línea]. Extraído el 24 de agosto del 2009 desde<http://www.uma.es/petra/proyectoteatinos/pu b/ATT00039.pdf>

9. Dejonckere, P.; Bradley, P.; Clemente, P.; Cornut, G.; Crevier- Buchman, L.; Friedrich, G. et. al. (2001). A basic protocol for functional assessment of voice pathology, especially for investigating the efficacy of phonosurgical treatments and evaluating new assessment techniques, Eur Arch Otorhinolaryngol, 258: 77-82.

10. Dikkers,F. (1994). Bening lesions of the vocal fold. Clinical and histopathological aspects, Tesis, Faculty of Medical Sciences, University of Groningen.
11. Núñez, F.; Corte, P.; Senqueiros, G.; Señaris, B. y Suárez, C. (2004). Evaluación perceptual de la disfonía: correlación con los parámetros acústicos y fiabilidad. Acta Otorrinolaringológica Española, 282-287.

12. Le Huche, F. y Allali, A. (2003). La Voz. Tomo IV. (2ª ed.). Barcelona: Elsevier. 